\title{
Omentum transposition for treatment of Alzheimer disease in China
}

\author{
Harry S. Goldsmith ${ }^{1,2}$
}

\begin{abstract}
Background: A paper was published in a Chinese medical journal that suggested that the placing of an intact vascularized omental pedicle would increase cerebral blood flow and could decelerate the development of Alzheimer's disease. However, the paper showed limited support for the operation. The purpose of this paper is to suggest that the lack of enthusiasm for the operation may deserve re-evaluation.

Method: The operation that was evaluated required an intact omental pedicle to be placed directly on the brain in order to increase the blood flow to the brain. The omentum is brought from the abdominal cavity up to the brain where it is simply laid resulting in a large volume of blood to flow into the cerebral tissue.

Results: The Chinese paper demonstrated relatively poor results in the Alzheimer patients. It is believed that the poor results were probably due to the results of the patients age, the length of time they had Alzheimer disease (5 or more years), but especially their low mini-mental state examination results - some as low as 2.

Conclusion: The paper being presented will suggest the possible benefit of omental transposition in Alzheimer patients when there is a successful choice of suitable candidates for the procedure.
\end{abstract}

Keywords: Omentum, Alzheimer's disease, Deteriorating neurons

\section{Omentum transposition to treat Alzheimer's disease in China}

In 2007, the Journal of the Shanghai $2^{\text {nd }}$ Medical University published a paper entitled, "Preliminary Study of Omentum Transposition to Brain for Treatment of Alzheimer's Disease" [1]. The main authors were Zhong $\mathrm{J}$ and $\mathrm{Wu} \mathrm{WL}$. I was graciously included as an additional author as I had participated in the cases reported in the publication. I was unaware, however, of the article and only learned of its existence years after its publication.

The authors concluded that placing the omentum on the brain of Alzheimer patients could increase cerebral blood flow (CBF) and could decelerate the development of Alzheimer disease (AD). They cautioned however, that the procedure is "potentially risky for the elderly." The apparent lack of enthusiasm for the operation has had a chilling effect on Chinese general surgeons/neurosurgeons who might consider performing omental transposition (OT) to the AD brain. The lack of support for the

Correspondence: hlgldsmith@aol.com

${ }^{1}$ University of California, Davis, USA

${ }^{2}$ Neurological Surgery, P.O. Box 493, Glenbrook, NV 89413, USA procedure should be more clearly evaluated since the operation has been shown to produce cognitive improvement in humans [2,3].

\section{Physiological considerations}

The decrease in cerebral blood flow in AD has been confirmed by spin-related magnetic resonance imaging and clearly relates the marked decrease in CBF as compared to age-matched controls [4]. This decrease in $\mathrm{CBF}$ in $\mathrm{AD}$ critically affects a host of physiological conditions in the brain such as oxygenation, lower glucose levels and an associated decrease in biological agents that normally are delivered to the brain by way of the bloodstream. This decrease in CBF is especially deleterious to the survival of cerebral neurons.

It has been commonly believed over the years that the decrease in $\mathrm{CBF}$ in $\mathrm{AD}$ was due to dead and dying neurons that no longer required a sustainable amount of CBF. However, this CBF decrease in AD may not be due to dying neurons that require less blood, but rather the $\mathrm{CBF}$ decrease is the result of normal aging, high blood 
pressure, diabetes and other factors that eventually lead to the death of neurons resulting in the cognitive deficiency observed in AD.

\section{Deteriorating neurons}

If one considers the possibility that $A D$ could be caused by a decrease in loss of cerebral blood flow, what would this effect have on neurons in an AD brain? There are three types of neurons in the $\mathrm{AD}$ brain - normal neurons; dead neurons that are the hallmark of $\mathrm{AD}$; and deteriorating neurons that are viable in different stages of dying [5]. It seems reasonable to expect that the greater the number of deteriorating neurons present in an $\mathrm{AD}$ brain, the earlier is the stage of $\mathrm{AD}$. Conversely, the fewer the number of deteriorating neurons present in the later stage of $\mathrm{AD}$, the greater the number of dead neurons that would be present. It would follow therefore, that any form of treatment of AD should be initiated at the earliest stage of $\mathrm{AD}$ when there would be a preponderance of viable deteriorating neurons.

It has been observed that in the entorinal cortex, in the early stages of $\mathrm{AD}, 60 \%$ of the neurons at the lamina 2 level are of the deteriorating type, and at the lamina 4 level, $40 \%$ of the neurons are of the deteriorating type [6]. The presence of such numbers of viable neurons in the entorinal cortex is important since the entorinal cortex is a critical cognitive location in the brain which is commonly damaged in $\mathrm{AD}$. Increasing $\mathrm{CBF}$ to the deteriorating but still viable neurons early in $\mathrm{AD}$ may explain the improved cognition that has been observed in $\mathrm{AD}$ patients [7].

\section{Neuronal survival}

When neurons involved in cognition experience a decrease in cerebral blood flow, their biological activity is adversely affected. This is true in the intraneuronal energy system that operates within cerebral neurons, especially on the effect of a decrease in CBF to intra-neuronal mitochondria. Mitochondria has a direct effect on the production of adenosine triphosphate (ATP), that is the energy source for neurons. Any reduction of ATP levels caused by a decrease in CBF has a negative effect on intracellular protein metabolism resulting in an accumulation of intracellular-extracellular amyloid $\mathrm{B}(\mathrm{AB})$ peptide in the $\mathrm{AD}$ brain [8]. When neurons in specific and critical areas of the brain (such as the hippocampus) begin to lose their cellular energy because of decreased ATP, an increase of CBF would likely benefit these neurons because of increased intra-neuronal mitochondrial activity, which would stimulate ATP production, the energy source for a neuron.

\section{Alzheimer's disease evaluation}

When one is considering surgery for an $\mathrm{AD}$ patient, it is important that the patient be thoroughly evaluated physically and cognitively. Surgery should not be performed simply because the patient has AD. An impartial medical person should evaluate the physical condition of the patient to determine whether the patient is a suitable candidate for surgery. If the degree of $\mathrm{AD}$ is in an early stage, a favorable outcome should be expected. Conversely, if a patient is in the late stages of $A D$, the expectation of post-operative success is less favorable. Additionally, if the patient is over 80 , a favorable outcome is less likely.

\section{Mini Mental State Examination (MMSE)}

The MMSE psychological test is an excellent way to determine the loss of cognitive awareness in AD. Personal experience has suggested that a high MMSE score raises expectation that $\mathrm{OT}$ to the $\mathrm{AD}$ brain may improve cognition. This is based on an apparent connection between MMSE levels in AD patients and the number of deteriorating neurons present in an AD brain. The MMSE test is based on a $1-30$ scale. Twenty to thirty on an MMSE scale indicates early AD; $16-20$ on the scale indicates moderately severe AD, and MMSE levels 15 or lower indicates a severe state of $\mathrm{AD}$. The connection between MMSE levels and the number of deteriorating neurons in an $A D$ brain is strongly suggested by the results of the patients in the Chinese Paper.

\section{Alzheimer's disease treatment}

There is presently no pharmaceutical agent that can increase cerebral blood flow for an extended period. There is however, a reported surgical procedure that can greatly increase $\mathrm{CBF}$. The operation involves placing the omentum on the brain (called omentum transposition OT) in which the omentum is lengthened and brought subcutaneously up the chest and neck to the head. A craniotomy is performed and the omentum is simply laid directly on the brain. No anastomosis is involved and there is direct penetration of a large volume of blood which flows from the omentum directly into the brain [9].

Despite statements of proof that patients with $\mathrm{AD}$ showed cognitive improvement following the surgery [10], there is a belief held by some that AD is incurable. This is based on the belief that AD is "irreversible because neurons that formally controlled cognitive function are largely dead and cannot be brought back to life" [11]. Obviously dead neurons cannot be revived, but if large numbers of viable deteriorating neurons are still present at the time of OT, increased CBF may explain why some patients have cognitively improved following OT to their brain. 


\section{Surgery}

The procedure of omentum transposition (OT) requires a laparotomy and a craniotomy. The patient is placed in a supine position with his head turned to either left or right depending on which side of the brain is to be covered by the omentum. An upper midline abdominal incision is made followed by the separation of the omentum from its proximal and central attachments to the stomach. Care must be taken that damage does not occur to the gastro-epiploic artery that remains within the omental pedicle. After the omental pedicle has been lengthed so that it can reach the top of the patients head, several small transverse incisions are made along the chest wall. These incisions are connected subcutaneously creating a long tunnel that extends from the upper pole of the mid-line abdominal incision up the chest wall and neck to behind the ear.

While the omental lengthening process is being developed, a temporal-parietal craniotomy is simultaneously carried out. The dura is opened and the omentum is placed directly on the brain. The omentum is then spread over the exposed brain area with the edges of the dura being secured to the omentum. The bone flap of the craniotomy is returned and the skin of the scalp is closed in a routine fashion.

\section{Discussion}

Although the Chinese Paper [1] indicates that age and physical health must be considered if a patient is suitable for surgery, one must question whether the patients in the Chinese study were suitable for a surgical procedure. The paper stated that all the patients had suffered from AD for more than five years, a time period that would suggest that the brains of these patients had a scarcity of deteriorating cerebral neurons and that the majority of their critical cerebral neurons were dead. The majority of the patients showed a post-operative increase in their cerebral blood flow, but the CBF increase would have had no effect on their non-viable cerebral neurons.

The Chinese patients were operated on because they had Alzheimer's disease. The importance of their MMSE scores was not considered. At the time they underwent surgery, no thought was raised as to the possible relationship between the number of deteriorating neurons in their brain and the patients MMSE score.

Information taken from the Chinese paper records the post-operative results of the patients. Their low MMSE scores, some as low as 2 , would raise the question as to whether post-operative success in some of the patients could have been expected. The paucity of deteriorating neurons in low MMSE patients who had suffered from $\mathrm{AD}$ over a 5-year period may well explain the lack of better post-operative result.
The information in the Chinese paper appears to substantiate a relationship between the number of deteriorating neurons and dead neurons found in the AD brain. This observation strongly suggests the importance of performing omentum transposition to the AD brain on patients as early as possible and only when their MMSE score is above 15-16.

\section{Conclusion}

It is known that millions of Chinese patients suffer from $\mathrm{AD}$ and this number rises on a daily basis. If numerous general and neuro-surgeons throughout China screen, evaluate and select those patients who would likely benefit from OT, it would be expected that favorable cognitive results will result.

Received: 8 April 2016 Accepted: 29 September 2016

Published online: 10 October 2016

References

1. Zhong J, WU WL. Preliminary study of omentum transposition to brain for treatment of Alzheimer's disease. J Shanghai Med Univ. 2007;19(1):46-51.

2. Shankle WR, Hara J, Bjornsen J, Gade GF, Leport PC, Ali MB, et al. Omentum transposition surgery for patients with Alzheimer disease: A case series. Neurol Res. 2008;30:313-25.

3. Shankle WR, Hara J, Bjornsen J, Gade GF, Leport PC, Ali MB, et al. Omental therapy for primary progressive aphasia with tau negative histopathology: 3 year study. Neurol Res. 2009;31:766-9.

4. Alsop DC, Derre JA, Grossman M. Assessment of cerebral blood flow in Alzheimer's disease by spin-labeled magnetic response imaging. Ann Neurol. 2000;47:93-100.

5. Goldsmith HS. Benefit of omental blood flow in Alzheimer disease: effect on deteriorating neurons. J Alzheimer Dis. 2014:42:5277-80.

6. Gomez-Isla TI, Price JL, McKeel Jr DW, Morris JC, Growden JH, Hyman BT. Profound loss of layer 2 entorinal cortex neurons occurs in very mild Alzheimer's disease. J Neurosci. 1996;16:4491-500.

7. Goldsmith HS. A new approach to the treatment o Alzheimer's disease. The need for a controlled study. J Alzheimer Dis. 2011;25:209-12.

8. de la Torre JC. Cerebral hypo-perfusion, capillary degeneration and development o Alzheimer disease. Alzheimer Dis Assoc Disord. 2000;14:573-81.

9. Goldsmith HS, Bacciu P, Cossu M, Pau A, Rodriguez G, Rosadini G, et al. Regional cerebral blood flow after omental transposition to the ischemic brain in man: A five year follow-up study. Acta Neurochir. 1990;106:145-52.

10. Goldsmith HS. Omental transposition in treatment of Alzheimer disease. J Am Coll Surg. 2007;205:800-4.

11. de la Torre JC. Alzheimer disease is incurable but preventable. J Alzheimer dis. 2010;20:861-70

Submit your next manuscript to BioMed Central and we will help you at every step:

- We accept pre-submission inquiries

- Our selector tool helps you to find the most relevant journal

- We provide round the clock customer support

- Convenient online submission

- Thorough peer review

- Inclusion in PubMed and all major indexing services

- Maximum visibility for your research

Submit your manuscript at www.biomedcentral.com/submit 